\title{
On the quest for selective constraints shaping the expressivity of the genes casting retropseudogenes in human
}

\author{
Kamalika Sen, Soumita Podder and Tapash C Ghosh
}

\begin{abstract}
Background: Pseudogenes, the nonfunctional homologues of functional genes are now coming to light as important resources regarding the study of human protein evolution. Processed pseudogenes arising by reverse transcription and reinsertion can provide molecular record on the dynamics and evolution of genomes. Researches on the progenitors of human processed pseudogenes delved out their highly expressed and evolutionarily conserved characters. They are reported to be short and GC-poor indicating their high efficiency for retrotransposition. In this article we focused on their high expressivity and explored the factors contributing for that and their relevance in the milieu of protein sequence evolution.

Results: We here, analyzed the high expressivity of these genes configuring processed or retropseudogenes by their immense connectivity in protein-protein interaction network, an inclination towards alternative splicing mechanism, a lower rate of mRNA disintegration and a slower evolutionary rate. While the unusual trend of the upraised disorder in contrast with the high expressivity of the proteins encoded by processed pseudogene ancestors is accredited by a predominance of hub-protein encoding genes, a high propensity of repeat sequence containing genes, elevated protein stability and the functional constraint to perform the transcription regulatory jobs. Linear regression analysis demonstrates mRNA decay rate and protein intrinsic disorder as the influential factors controlling the expressivity of these retropseudogene ancestors while the latter one is found to have the most significant regulatory power.

Conclusions: Our findings imply that, the affluence of disordered regions elevating the network attachment to be involved in important cellular assignments and the stability in transcriptional level are acting as the prevailing forces behind the high expressivity of the human genes configuring processed pseudogenes.
\end{abstract}

Keywords: Expressivity, Protein intrinsic disorder, Connectivity, Alternative splicing, Protein stability, mRNA decay rate, Evolutionary rate

\section{Background}

Pseudogenes often exemplified as 'genetic fossils' provide snapshots of evolutionary history of human genome [1]. Understanding the structural and functional attributes of the genes configuring pseudogenes by duplication and reverse transcription is now enlightening the research on these naturally occurring mutant genes in the frame of evolutionary studies supporting neutral mutation hypotheses [2]. The occurrence of these faulty

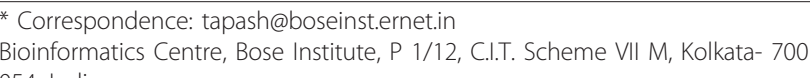

* Correspondence: tapash@boseinst.ernet.in
Bioinformatics Centre, Bose Institute, P 1/12, C.I.T. Scheme VII M, Kolkata- 700 054, India
}

(c) 2011 Sen et al; licensee BioMed Central Ltd. This is an Open Access article distributed under the terms of the Creative Commons Biomed Central Attribution License (http://creativecommons.org/licenses/by/2.0), which permits unrestricted use, distribution, and reproduction in any medium, provided the original work is properly cited.

replicates of normal genes in a genome is still a confounding matter. The processed or retropseudogenes [3] speculated as fossilized footprints of their parental gene expression [4] has become of increasing interest in the field of pseudogene evolution and comparative genomics since a burst of processed pseudogene genesis was observed early in primate evolution [5]. This kind of pseudogenes resembles the mature mRNA transcript of their functional counterpart. The processed transcript of a functional gene is reverse transcribed and integrated into a staggered chromosome break, followed by DNA synthesis and repair [6]. The process of reverse 
transcription and insertion is guided by the enzymatic machinery of LINE1 non-LTR retrotransposons [7]. Being derived from a mature mRNA product they lack the upstream promoters and are often entitled as "dead on arrival" [8] because of their acquired nonfunctionality [9] immediately upon the reinsertion process. Their structural feature shows a complete lack of introns, small flanking direct repeats and polyadenilation at the 3'-end [10].

During the last several years processed pseudogenes are being catalogued and characterized in many completely sequenced genomes including human. But there are very few reports on the structural and functional characterization of the human genes configuring this kind of pseudogenes. The pioneering work of Goncalves et al [11] focused on 181 human functional genes giving rise to 249 retropseudogenes. Their analysis revealed out some important features of the genes generating retropseudogenes regarding their evolutionary impact and structural attributes. They reported them (genes with retropseudogenes) to execute a significantly higher value of tissue distribution breadth than the genes lacking retropseudogenes. The preponderance of processed pseudogenes in housekeeping genes is also relevant to their higher expression level [12] than the genes without pseudogenes. Again, expression of a gene has been considered as a crucial marker of the evolutionary perseverance of the same till date [13]. So in the context of human processed pseudogene ancestor evolution gene expression and its different facets surely craves an intensive attention and a comprehensive discussion.

In this communication we probed the different aspects of gene expression of the human processed pseudogene ancestors. We characterized the high expressivity of the progenitor genes by their involvement in protein-protein interaction network, affluence of intrinsically unstructured protein regions, selection for alternative splicing technique, transcript stability and evolutionary conservation.

\section{Results}

Expression profile of the progenitor of processed pseudogenes and their involvement in protein-protein interaction network

Earlier it was reported that retropseudogene ancestors are predominantly housekeeping in nature showing a wide tissue distribution breadth [11]. The ubiquitously expressed housekeeping genes are also seen to execute high gene expression level [14]. We thus verified the high expressivity of the progenitors of processed pseudogenes (GFP $\psi$ genes) which are known to belong to housekeeping gene class. In our search the processed pseudogene ancestors exhibited significantly higher signal intensity $\left(\mathrm{P}=1.61 \times 10^{-86}\right.$ in Mann-Whitney test
(M-W test), average expression level 2157.7995 [GFP $\psi$ genes], 961.2597 [GL $\psi$ genes]) as well as a higher EST count $\left(\mathrm{P}=3.26 \times 10^{-136}\right.$ in $\mathrm{M}-\mathrm{W}$ test, average values $40.996[\mathrm{GFP} \psi$ genes], 18.202 [GL $\psi$ genes]) expressing the mRNA abundance than the genes lacking pseudogenes. Now connectivity in interaction network and the gene expression level have been reported to be elevated for the genes belonging to housekeeping class [15]. Comparing the network attachment of the gene groups we observed a significantly higher value of interacting partners $\left(\mathrm{P}=5.0 \times 10^{-3}\right.$ in $\mathrm{M}-\mathrm{W}$ test, average connectivity $8.3638[\mathrm{GFP} \psi$ genes], 7.6434 [GL $\psi$ genes]) for the genes giving rise to processed pseudogenes than the genes with no pseudogenes. A significant positive correlation linking connectivity (between interacting partners) and signal intensity as well as connectivity and mRNA abundance was also obtained in our study (Table-1). Previous studies revealed that genes encoding hub proteins tend to be expressed with higher intensity [16]. Owing to this fact we also checked out the propensity of hub-protein encoding genes in our dataset and there we observed a significant predominance $(Z$ score $=$ 3.842, confidence level $=99 \%$ ) of hub-protein encoding genes in the GFP $\psi$ genes (41.70\%) than that of the GL $\psi$ genes (35.58\%).

\section{Predominance of disordered residues in the protein} sequences of the genes configuring retropseudogenes Proteins expressed at higher levels are stated to contain less disordered regions [17]. Hence, the ancestor genes of the processed pseudogenes which are seen to be highly expressed in our study were expected to show a low content of disordered residues. But surprisingly, in our analysis the progenitors of processed pseudogenes displayed a significantly higher amount of disordered residues $\left(\mathrm{P}=6.56 \times 10^{-26}\right.$ in $\mathrm{M}-\mathrm{W}$ test, average disordered residue 41.924\% [GFP $\psi$ genes], 32.991\% [GL $\psi$ genes]) than the genes without pseudogenes and the percentage of disordered residues executed a significant positive association with the expression level of the two gene groups concerned (Table-1). To resolve this contradiction, we concentrated on the arguments which

Table 1 Spearman's Rho and P values of the statistical correlations between the parameters analyzed in GFP $\psi$ genes and GL $\psi$ genes

\begin{tabular}{lllll}
\hline Parameters & \multicolumn{2}{l}{$\begin{array}{l}\text { Gene expression } \\
\text { (using Microarray) }\end{array}$} & \multicolumn{2}{l}{$\begin{array}{l}\text { Gene expression } \\
\text { (using EST) }\end{array}$} \\
\hline & $\boldsymbol{\rho}$ & $\mathbf{P}$ & $\boldsymbol{\rho}$ & $\mathbf{P}$ \\
\hline Interacting partners & 0.145 & $1.0 \times 10^{-6}$ & 0.121 & $1.0 \times 10^{-6}$ \\
\hline Protein intrinsic disorder & 0.058 & $1.84 \times 10^{-6}$ & 0.039 & $2.94 \times 10^{-4}$ \\
\hline mRNA decay rate & -0.118 & $1.0 \times 10^{-6}$ & -0.119 & $1.0 \times 10^{-6}$ \\
\hline Evolutionary rate & -0.133 & $1.0 \times 10^{-6}$ & -0.137 & $1.0 \times 10^{-6}$ \\
\hline
\end{tabular}


claimed that, the highly expressed proteins engaged in binding functions employ disordered regions to prevent aggregation [17] and the presence of unstructured regions is a constitutive feature of the hub-proteins since the disorder can act as a determinant of protein interactivity [18]. So, the GFP $\psi$ genes with higher expression level exhibited an elevated disorder due to their intense connectivity and higher propensity of hubprotein forming genes. To endorse our observations regarding the protein disorder we looked into other genomic and functional features of the retropseudoegene ancestors dealing with protein unfolded ness.

Earlier studies revealed an enrichment of disorder producing residues in highly stable proteins $[19,20]$ due to the possibility that in vivo the disordered regions are no longer "unstructured" and are protected by binding to their biological targets. In our search the progenitors of the processed pseudogenes exhibit a significantly higher value of protein stability index $\left(\mathrm{P}=2.40 \times 10^{-8}\right.$ in $\mathrm{M}-\mathrm{W}$ test, average value 3.658 [GFP $\psi$ genes], 3.390 [GL $\psi$ genes]) over the genes without pseudogenes.

Along with the protein stability and network involvement, we also probed into the genomic and functional features of the GFP $\psi$ genes to demonstrate their high disorder. Tandem repeat regions are seen to be prevalent in intrinsically unstructured regions [21]. We thus analyzed the presence of tandem repeat sequences in the human genes giving rise to processed pseudogenes which are affluent with disordered regions. Analyses revealed a significantly higher propensity $(Z$ score $=2.558$, confidence level $=95 \%)$ of genes having tandem repeat sequences in the aforementioned gene pool compared to that of the gene group lacking pseudogenes.

Researchers also provided evidence for the fact that proteins which are entirely disordered (80\% to $100 \%$ disordered residue) with high level of expression can bypass the route of rapid degradation to successfully carry out their functions [19]. This kind of genes typically works as parts of large ribosomal subunits involved in transcription machinery [19]. In our experimentation, the genes with $80 \%-100 \%$ disordered residues and representing large ribosomal subunits engaged in transcription are significantly predominant $(Z$ score $=9.819$, confidence level $=99 \%, 11.28 \%$ [GFP $\psi$ genes], 4.16\% [GL $\psi$ genes] and $Z$ score $=13.05$, confidence level 99\%, 9.48\% [GFP $\psi$ genes], 3.91\% [GL $\psi$ genes] respectively) in the gene pool of the processed pseudogene ancestors than the pseudogene lacking gene set. Thus the processed pseudogene ancestors simultaneously exhibiting high expressivity and high disorder ness belong to a typical class of genes having the functional constraint to perform transcription associated works.
The progenitors of processed pseudogenes produce a large number of spliced isoforms and execute a high level of mRNA stability

Studies on genes with disordered residues revealed that alternative splicing sites are prevalent within the regions which are intrinsically unstructured [22]. Again it was established that alternative splicing can modulate gene expression by controlling transcript stability and translational efficacy [23]. In our search the genes with natively unfolded regions, giving rise to retropseudogenes are found to produce a number of spliced isoforms. We observed the number of those spliced isoforms to be significantly greater $\left(\mathrm{P}=3.38 \times 10^{-4}\right.$ in $\mathrm{M}-\mathrm{W}$ test, average spliced isoform number $5.96[\mathrm{GFP} \psi$ genes], $5.75[\mathrm{GL} \psi$ genes] and respectively) in the aforementioned gene group than that of the genes lacking pseudogenes. There is also a significant positive correlation (Spearman's $\rho=0.357, \mathrm{P}=1.0 \times 10^{-6}$ ) with the number of splicing isoforms and the mRNA abundance in the $\mathrm{GFP} \psi$ gene group (Figure 1).

Moreover, it was argued that, abundant mRNAs are likely to be considered as substrate of reverse-transcriptase enzyme [11]. As a consequence of the increased probability of reverse transcription process the chance for retro-pseudogenization event may also get raised which is reflected in our result obtained for GFP $\psi$ genes where we got a significantly positive correlation (Spearman's $\rho=0.231, \mathrm{P}=1 \times 10^{-6}$ ) between mRNA abundance and the number of processed pseudogenes per ancestor gene (Figure 2).

While treating gene expression level, we again looked into the issue where it was stated that a higher rate of mRNA decay can be considered as an indicator of the lower gene expressivity [19]. In addition, the translational robustness of the genes configuring retroposed human mRNAs is also explained by their resistance to nonsense-mediated RNA decay [24]. Here, in our

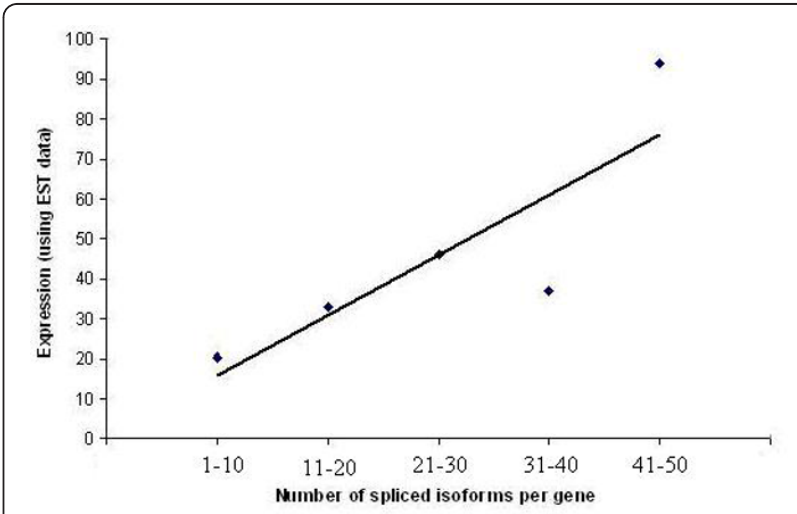

Figure 1 Scatter plot showing correlation between number of spliced isoforms per gene and expression (using EST data). 


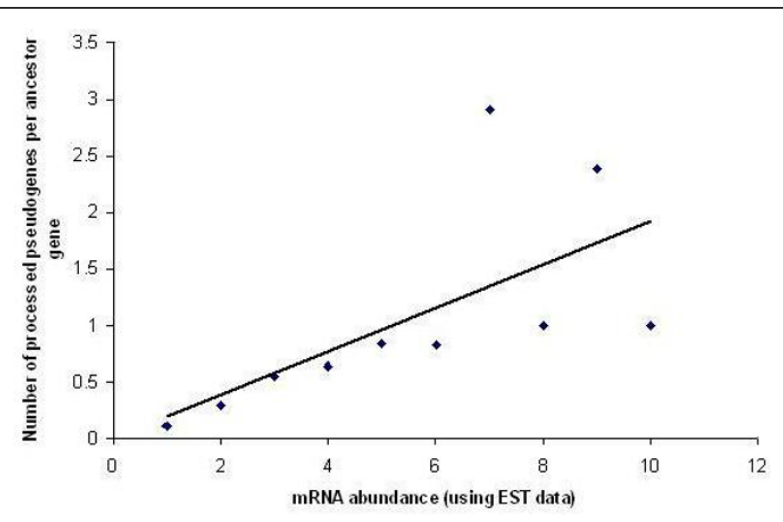

Figure 2 Scatter plot showing correlation between mRNA abundance and number of processed pseudogenes per ancestor gene. The EST data is clustered into 10 bins having a regular increase of mRNA abundance number 25 .

analysis, the genes promoting processed pseudogenes exhibit a coherence of higher expressivity and lower mRNA decay rate. We observed a significantly higher mRNA decay rate $\left(\mathrm{P}=1.21 \times 10^{-6}\right.$ in $\mathrm{M}-\mathrm{W}$ test, $1.479 \times$ $10^{-1}$ [GL $\psi$ genes], $1.094 \times 10^{-1}$ [GFP $\psi$ genes]) of the genes without pseudogenes than the genes casting processed pseudogenes along with a significant negative correlation associating the mRNA decay rate and gene expression level (Table-1). Thus the mRNA stability of the GFP $\psi$ genes also account for their higher expressivity.

\section{Amino-acid sequence conservation of the ancestors of processed pseudogenes}

Evolutionary studies on pseudogenes revealed that they congregate mutations at an extremely higher rate uniformly over their entirety when compared with their functional counterpart [25]. In our previous report on the duplicated pseudogenes we observed their ancestor genes to evolve at a higher rate than the genes configuring functional genes [26]. On the other hand, it has been reported that, genes with retropseudogenes encounter a stronger selective pressure for amino acid sequence conservation than the genes without retropseudogenes [11]. Besides, the rate of evolution is observed to be modulated by the intensity of gene expression of vertebrate genome [27]. Even in yeast the highly expressed genes are seen to evolve slowly [28]. These observations induced us to have a comparative look into the pattern of evolutionary rate of the GFP $\psi$ genes and the GL $\psi$ genes and to justify the relation with gene expression level. Our results showing a significantly lower rate of evolution $\left(\mathrm{P}=8.1 \times 10^{-11}\right.$ in $\mathrm{M}-\mathrm{W}$ test, $1.378 \times 10^{-1}[\mathrm{GL} \psi$ genes $], 1.261 \times 10^{-1}[\mathrm{GFP} \psi$ genes $\left.]\right)$ for the progenitors of processed pseudogenes than the genes without pseudogenes along with a significant negative correlation between gene expression and rate of evolution (Table-1) confirm the above mentioned facts.

\section{Discussion}

Previous reports unveiling the housekeeping character and evolutionary persistence of the progenitors of processed pseudogenes demand further delving on their pattern of gene expression. Our investigations on gene expression level revealed a high expressivity of the human genes casting processed pseudogenes over the genes lacking pseudogenes. We thereafter tried to scoop out the genomic and functional traits of the genes processing retropseudogenes to elucidate their high expressivity.

The intensity of protein expression and physical interactions are seen to be integrated in humans [29]. Reports also claimed a high expressivity for the genes well connected (hub-protein encoding genes) in proteinprotein interaction network [30]. In our study the high expressivity and wide tissue distribution breadth shaping the interaction pattern of the parent genes of processed pseudogenes in protein-protein interaction network reflect the same issue since they are found to execute high interactivity along with a predominance of hubprotein encoding genes. The disparity appeared in the results when we observed the retropseudogene ancestors with high gene expression displayed an affluence of disordered regions. We thence, demonstrated the disorderness as a prerequisite of their intense network involvement. The unstructured regions in their translated forms facilitate the network connectivity since previous reports demonstrated structural disorderness as a common characteristic of hub proteins [18]. The GFP $\psi$ genes harboring disordered regions and displaying a high expressivity and network connectivity are also observed to retain their translated form in a stable configuration. This may be due to the fact that, the regions with structural disorder keep up the stability of proteins in vivo through the attachment with their corresponding target molecules [20]. While evaluating the existence of disordered regions in the proteins encoded by the GFP $\psi$ genes we put forward more evidences providing their genomic and functional features. In doing so we delved into the fact that, hub-proteins are enriched with disordered residues and sequence repeats to enlarge available surface area predisposing them for functioning via protein-protein interactome [31]. In our research on the ancestors of retropseudogenes, the abundance of genes encoding hub-proteins and repeat sequences also affirms the occurrence for protein disordered regions. In addition, a positive correlation (Spearman's $\rho=0.071, \mathrm{P}=$ $1.0 \times 10^{-6}$ ) between the mRNA abundance and the propensity of repeat sequence containing gene supports the 
fact that, tandem repeats in human genes can positively regulate the level of transcription [32]. Moreover, proteins encoded by the GFP $\psi$ genes are observed to configure large ribosomal subunits engaged in transcription associated jobs. Our result thus provides support for the idea that, proteins carrying disordered regions are able to perform some essential functions directly linked to their structural disorder [33]. Thus, even the lack of welldefined 3-D structure of the proteins encrypted by the GFP $\psi$ genes also represents their significance and functional importance in human protein interactome. A positive correlation (Spearman's $\rho=0.09, \mathrm{P}=1.0 \times 10^{-6}$ ) observed between protein stability index and mRNA abundance further confirms the fact that, the highly disordered proteins can bypass rapid degradation pathway to allow them to perform their important cellular functions [19]. In addition, the analysis of the mRNA decay rates of the two gene group yields slower mRNA decay for the GFP $\psi$ genes supporting earlier reports which displayed mRNA turnover as a factor to coordinate gene expression level via transcriptional and translational regulation [19,34]. Again, it was argued that intrinsically unstructured regions of a polypeptide segment offer sites for alternative splicing as the disordered regions can tolerate functional or regulatory diversity without any disturbance in protein sequence [22]. Furthermore, as the alternative splicing event accounts for the quantitative and qualitative regulation of gene expression [24], we tried to explain the elevation in mRNA abundance (representing expression level of the gene groups) observed in the GFP $\psi$ genes in terms of their inclination towards alternative splicing mechanism. Hence, we here hypothesize that, the GFP $\psi$ genes go through an extensive alternative splicing event to form a number of spliced isoforms elevating the mRNA abundance level. The degree of mRNA abundance and their endurance together may contribute to enhance the level of gene expression of the GFP $\psi$ genes. We here, also speculate that the higher mRNA abundance contributes for an elevated reverse-transcription process which in turn increases the chance for retro-pseudogenization. Moreover, as gene expression level is known to constrain sequence evolution [35] in yeast, we here, analyzing human genes, also examined rate of protein evolution and observed amino acid sequence conservation for the genes promoting processed pseudogenes in human which again affirms their functional significance. Finally, from the linear regression analysis we can confer that, though all the factors we analyzed here control the expressivity of the GFP $\psi$ genes but all of them can not act as independent regulator of the same. It is evident from the analyses that, only the percentage of disordered residues $(\beta=$ $0.194)$ and the mRNA decay rate $(\beta=-0.079)$ can independently control the expressivity where the disorder influences the gene expression most significantly. We also performed our analysis excluding the ribosomal proteins and got the same trend in our results and confirmed that their (ribosomal proteins) evolutionary persistence, higher expression and higher disorder did not predispose the results (Additional file 1).

\section{Conclusions}

Taken together, we summarize that, the regulatory factors constraining the expressivity of the human genes configuring processed pseudogenes are their connectivity in protein-protein interaction network, alternative splicing mechanism, slower rate of mRNA decay and evolutionarily conserved nature. While, the presence of intrinsically unstructured regions, which is attributed by the occurrence of genes containing repeat sequences, increased protein stability and the functional constraint to perform the transcription regulatory jobs, enriches the network connectivity to be involved in important cellular assignments and increase the stability in transcriptional level. Thus, our study on the human processed pseudogene ancestors, exposing the genomic imperatives constraining their expressivity as well as a new facet of their physical and functional attributes will expand the future studies on pseudogene forming genes on the scaffold of human genome evolution.

\section{Methods}

\section{Human genes forming processed pseudogenes}

Human processed pseudogene annotations were achieved from pseudogene database (Human pseudogenes, Build 57) (http://www.pseudogene.org/) [36]. In our analysis the human genes forming processed pseudogenes are allocated as GFP $\psi$ genes and the genes without pseudogenes are assigned as GL $\psi$ genes. The number of genes retrieved as GFP $\psi$ genes and GL $\psi$ genes are 2362 and 38,862 respectively. The corresponding gene sequences were obtained from ftp://ftp.ncbi. nih.gov/genomes/H_sapiens/.

\section{Gene microarray expression data}

The gene expression profile data were extracted from Human Gene Atlas GNF1H, MAS5 dataset (http:// biogps.org/) [14]. The signal intensities across 79 tissues were averaged and are considered as expression level for each gene represented by their corresponding probe id. The number of genes retrieved as GFP $\psi$ genes and GL $\psi$ genes were 2362 and 38,862 respectively. However, we performed our other analyses with the genes providing Microarray expression intensity and the number of genes (in Microarray expression dataset) for GFP $\psi$ genes and GL $\psi$ genes are 1443 and 10192 respectively (Additional file 2). 
Protein-protein interaction data

The number of interacting partners of the genes in our dataset was obtained from HPRD (Human Protein Reference Database), version 7, (http://www.hprd.org) [37] and the genes maintaining more than 5 interacting partners were assigned as hub proteins.

\section{Disorder prediction in human proteome}

Disorder predictions were carried out using the program FoldIndex [38] implementing the prediction method of Uversky et al [39]. To reduce the rate of false positives, disordered regions containing at least 30 contiguous disordered residues were considered [40]. The fraction of disordered residues was calculated by taking the ratio of the number of disordered residues to the total number of residues in the protein.

\section{Measurement of protein stability indices}

Data for protein stability indices were obtained from the stability measures done by Yen et al [20] in their global protein stability assay of more than 8,000 human proteins. We mapped the stability measures to our gene dataset.

\section{Retrieval of repeat sequence containing genes}

Repeat regions of genes were found out using the program Tandem repeats finder [41]. The propensity of repeat region containing genes was calculated by considering the ratio of number of genes having repeat regions to the total number of genes in each dataset.

\section{Functional annotations of our gene sets}

The functional information assigned by the GO annotations of the highly disordered proteins was obtained from the Go term data of Edwards et al [19]. We mapped the given Go annotations on our dataset.

\section{Retrieval of alternatively spliced isoforms}

Data for alternatively spliced isoforms [42] for the genes in dataset was downloaded from the ASD database (http://www.ebi.ac.uk/asd) containing splice patterns of human alternatively spliced genes.

\section{Measurement of mRNA abundance}

mRNA abundance of the genes in our dataset was calculated using EST data attained from DFCI Gene Indices (http://compbio.dfci.harvard.edu/tgi/). Gene expression level was estimated calculating the number of occurrence of each gene among EST sequences from 179 cDNA libraries sampled with at least 10,000 ESTs $[43,44]$. Eliminating pathogenic and cancerous libraries 41 libraries were kept and alignments were made between the coding sequences of the gene groups with the EST dataset using BLASTN program with a sequence matching criterion of $60 \%$ identity and $80 \%$ overlaps. The overall EST count for each gene across 41 EST libraries represents their mRNA abundance.

\section{Evaluation of mRNA decay rates}

mRNA decay rates of the genes in our dataset were retrieved from the report/analysis of Yang et al [45] where they measured the mRNA decay rates of 5,245 human transcript.

\section{Data for gene evolutionary rates}

Evolutionary rates of the human genes (Homo sapiens (GRCh37)) in our dataset (genes with processed peudogenes and without pseudogenes) were achieved from Ensembl 58 (http://www.ensembl.org/biomart/martview) [46].

\section{Retrieval of genes encoding Ribosomal Proteins}

Genes coding ribosomal proteins were obtained from Ribosomal Protein Gene Database (http://ribosome. miyazaki-med.ac.jp) [47].

\section{Additional material}

Additional file 1: $\mathrm{P}$ values in Mann-Whitney tests for the comparative study performed between GFP $\psi$ genes and GL $\psi$ genes after removing all genes coding Ribosomal proteins from both the datasets.

Additional file 2: Ensmble id of Genes Forming Processed Pseudogenes (GFP $\psi$ genes) and Genes Lacking any Pseudogenes (GL $\psi$ genes).

\section{Abbreviations}

GFP $\Psi$ genes: Genes Forming Processed Pseudogenes; GL $\psi$ genes: Genes Lacking Pseudogenes.

\section{Acknowledgements}

Authors are thankful to the Department of Biotechnology, Govt. of India for financial help. We thank Mr. S.K. Gupta for his technical helps and Dr. Angshuman Bagchi for critical reading of the manuscript. We are also thankful to two anonymous referees for their helpful suggestions in improving the manuscript.

Authors' contributions

KS conceived of the study, collected the data, performed steps of bioinformatic analysis, statistical analysis and wrote the manuscript. SP contributed in the design of the study, collection of data and coordination. TCG supervised the study and helped to draft the manuscript. All authors read and approved the final manuscript.

Received: 31 December 2010 Accepted: 8 August 2011

Published: 8 August 2011

\section{References}

1. Zhang ZL, Harrison PM, Liu Y, Gerstein M: Millions of years of evolution preserved: A comprehensive catalog of the processed pseudogenes in the human genome. Genome Research 2003, 13(12):2541-2558.

2. Torrents D, Suyama M, Zdobnov E, Bor P: A Genome-Wide Survey of Human Pseudogenes. Genome Research 2003, 13:2559-2567. 
3. Cs"ur"os M, Mikl'os I: Statistical Alignment of Retropseudogenes and Their Functional Paralogs. Mol Biol Evol 2005, 22(12):2457-2471.

4. Podlaha O, Zhang JZ: Processed pseudogenes: the 'fossilized footprints' of past gene expression. Trends in Genetics 2009, 25(10):429-434.

5. Ohshima K, Hattori M, Yada T, Gojobori T, Sakaki Y, Okada N: Wholegenome screening indicates a possible burst of formation of processed pseudogenes and Alu repeats by particular L1 subfamilies in ancestral primates. Genome Biology 2003, 4:R74.

6. Vanin EF: Processed pseudogenes-characteristics and evolution. Annual Review of Genetics 1985, 19:253-272.

7. Esnault C, Maestre J, Heidmann T: Human LINE retrotransposons generate processed pseudogenes. Nature Genetics 2000, 24(4):363-367.

8. Ding WY, Lin L, Cheh B, Dai JW: L1 elements, processed pseudogenes and retrogenes in mammalian genomes. lubmb Life 2006, 58(12):677-685.

9. Graur D, Li W-H: Fundamentals of Molecular Evolution. Sunderland, Mass: Sinauer Associates, Inc; Second 2000.

10. Zhang ZL, Gerstein M: Large-scale analysis of pseudogenes in the human genome. Current Opinion in Genetics \& Development 2004, 14(4):328-335.

11. Goncalves I, Duret L, Mouchiroud D: Nature and structure of human genes that generate retropseudogenes. Genome Research 2000, 10(5):672-678

12. Guo X, Zhang Z, Gerstein MB, Zheng D: Small RNAs Originated from Pseudogenes: cis-or trans-Acting? Plos Computational Biology 2009, 5(7): e1000449.

13. Gout JF, Kahn D, Duret L, Paramecium Post-Genomics Consortium: The Relationship among Gene Expression, the Evolution of Gene Dosage, and the Rate of Protein Evolution. Plos Genetics 2010, 6(5)

14. Su Al, Wiltshire T, Batalov S, Lapp H, Ching KA, Block D, Zhang J, Soden R, Hayakawa M, Kreiman G, et al: A gene atlas of the mouse and human protein-encoding transcriptomes. Proceedings of the National Academy of Sciences of the United States of America 2004, 101(16):6062-6067.

15. Reverter A, Ingham A, Dalrymple BP: Mining tissue specificity, gene connectivity and disease association to reveal a set of genes that modify the action of disease causing genes. BioData Min 2008, 1(1):8.

16. Wu XD, Qi XQ: Genes encoding hub and bottleneck enzymes of the Arabidopsis metabolic network preferentially retain homeologs through whole genome duplication. Bmc Evolutionary Biology 2010, 10:

17. Singh GP, Dash D: How expression level influences the disorderness of proteins. Biochemical and Biophysical Research Communications 2008, 371(3):401-404.

18. Haynes C, Oldfield CJ, Ji F, Klitgord N, Cusick ME, Radivojac P, Uversky VN, Vidal M, lakoucheva LM: Intrinsic disorder is a common feature of hub proteins from four eukaryotic interactomes. Plos Computational Biology 2006, 2:890-901.

19. Edwards YJK, Lobley AE, Pentony MM, Jones DT: Insights into the regulation of intrinsically disordered proteins in the human proteome by analyzing sequence and gene expression data. Genome Biology 2009, 10(5)

20. Yen HCS, Xu QK, Chou DM, Zhao ZM, Elledge SJ: Global Protein Stability Profiling in Mammalian Cells. Science 2008, 322(5903):918-923.

21. Tompa P: Intrinsically unstructured proteins evolve by repeat expansion. Bioessays 2003, 25(9):847-855

22. Romero PR, Zaidi S, Fang YY, Uversky VN, Radivojac P, Oldfield CJ, Cortese MS, Sickmeier M, LeGall T, Obradovic Z, et al: Alternative splicing in concert with protein intrinsic disorder enables increased functional diversity in multicellular organisms. Proceedings of the National Academy of Sciences of the United States of America 2006, 103(22):8390-8395.

23. Smith CWJ, Patton JG, Nadalginard B: Alternative splicing in the control of gene-expression. Annual Review of Genetics 1989, 23:527-577.

24. Pavlicek A, Gentles AJ, Pačes J, Pačes V, Jurka J: Retroposition of processed pseudogenes: the impact of RNA stability and translational control. Trends in Genetics 2006, 22:69-73.

25. Miyata T, Hayashida H: Extraordinarily high evolutionary rate of pseudogenes-evidence for the presence of selective pressure against changes between synonymous codons. Proceedings of the National Academy of Sciences of the United States of America-Biological Sciences 1981, 78(9):5739-5743.

26. Sen K, Podder S, Ghosh TC: Insights into the genomic features and evolutionary impact of the genes configuring duplicated pseudogenes in human. Febs Letters 2010, 584(18):4015-4018.
27. Subramanian S, Kumar S: Gene expression intensity shapes evolutionary rates of the proteins encoded by the vertebrate genome. Genetics 2004, 168(1):373-381.

28. Pal C, Papp B, Hurst LD: Highly expressed genes in yeast evolve slowly. Genetics 2001, 158(2):927-931

29. Bossi A, Lehner B: Tissue specificity and the human protein interaction network. Molecular Systems Biology 2009, 5.

30. Batada NN, Hurst LD, Tyers M: Evolutionary and physiological importance of hub proteins. Plos Computational Biology 2006, 2(7):748-756.

31. Dosztanyi Z, Chen J, Dunker AK, Simon I, Tompa P: Disorder and sequence repeats in hub proteins and their implications for network evolution. Journal of Proteome Research 2006, 5:2985-2995.

32. Gemayel R, Vinces MD, Legendre M, Verstrepen KJ: Variable Tandem Repeats Accelerate Evolution of Coding and Regulatory Sequences. Annu Rev Genet 2010, 44:445-77.

33. Hazy E, Tompa P: Limitations of Induced Folding in Molecular Recognition by Intrinsically Disordered Proteins. ChemPhysChem 2009, 10:1415-1419.

34. Wilusz CJ, Wilusz J: Bringing the role of rnRNA decay in the control of gene expression into focus. Trends in Genetics 2004, 20(10):491-497.

35. Drummond DA, Bloom JD, Adami C, Wilke CO, Arnold FH: Why highly expressed proteins evolve slowly. Proceedings of the National Academy of Sciences of the United States of America 2005, 102(40):14338-14343.

36. Karro OE, Yan Y, Zheng D, Zhang Z, Carriero N, Cayting P, Harrison P, Gerstein M: Pseudogene.org: a comprehensive database and comparison platform for pseudogene annotation. Nucleic Acids Research 2007, 35 D55-60.

37. Prasad TSK, Goel R, Kandasamy K, Keerthikumar S, Kumar S, Mathivanan S, Telikicherla D, Raju R, Shafreen B, Venugopal A, et al: Human Protein Reference Database-2009 update. Nucleic Acids Research 2009, 37: D767-D772.

38. Prilusky J, Felder CE, Zeev-Ben-Mordehai T, Rydberg EH, Man O, Beckmann JS, Silman I, Sussman JL: Foldlndex(c): a simple tool to predict whether a given protein sequence is intrinsically unfolded. Bioinformatics 2005, 21(16):3435-3438.

39. Uversky VN, Gillespie JR, Fink AL: Why are "natively unfolded" proteins unstructured under physiologic conditions? Proteins-Structure Function and Genetics 2000, 41(3):415-427.

40. Obradovic Z, Peng K, Vucetic S, Radivojac P, Brown CJ, Dunker AK: Predicting intrinsic disorder from amino acid sequence. Proteins-Structure Function and Genetics 2003, 53(6):566-572.

41. Benson G: Tandem repeats finder: a program to analyze DNA sequences. Nucleic Acids Research 1999, 27(2):573-580.

42. Stamm S, Riethoven JJ, Le Texier V, Gopalakrishnan C, Kumanduri V, Tang YS, Barbosa-Morais NL, Thanaraj TA: ASD: a bioinformatics resource on alternative splicing. Nucleic Acids Research 2006, 34:D46-D55.

43. Podder S, Mukhopadhyay P, Ghosh TC: Multifunctionality dominantly determines the rate of human housekeeping and tissue specific interacting protein evolution. Gene 2009, 439(1-2):11-24

44. Duret L, Mouchiroud D: Expression pattern and, surprisingly, gene length shape codon usage in Caenorhabditis, Drosophila, Arabidopsis. Proceedings of the National Academy of Sciences of the United States of America 1999, 96(8):4482-4487.

45. Yang E, van Nimwegen E, Zavolan M, Rajewsky N, Schroeder M, Magnasco M, Darnell JE: Decay rates of human mRNAs: Correlation with functional characteristics and sequence attributes. Genome Research 2003, 13(8):1863-1872

46. Hubbard TJP, Aken BL, Ayling S, Ballester B, Beal K, Bragin E, Brent S, Chen Y, Clapham P, Clarke L, et al: Ensembl 2009. Nucleic Acids Research 2009, 37:D690-D697.

47. Nakao A, Yoshihama A, Kenmochi N: RPG: The Ribosomal Protein Gene database. Nucleic Acids Research 2004, 32:D168-D170.

doi:10.1186/1471-2164-12-401

Cite this article as: Sen et al:: On the quest for selective constraints shaping the expressivity of the genes casting retropseudogenes in human. BMC Genomics 2011 12:401. 